\title{
Use of Point-of-Care Ultrasonography in Primary Care to Redress Health Inequities
}

\author{
Michael Tanael, MD
}

( J Am Board Fam Med 2021;34:853-855.)

Keywords: Family Medicine, Health Care Disparities, Outcomes Assessment, Point-of-Care Systems, Primary Health Care, Social Determinants of Health, Ultrasonography

A growing body of literature has raised awareness of avoidable and unjust demographic differences in health outcomes in America. Many proposals to mitigate these differences-these health inequities -leverage technologic advancements to improve care, but few highlight the potential benefits of using point-of-care ultrasonography (POCUS) by primary care clinicians. ${ }^{1}$ The expanded capabilities this technology provides outpatient generalists allow them to circumvent economic, social, educational, health care, and environmental conditions that produce health inequities.

Several recent narrative reviews outline applications of POCUS that primary care clinicians can incorporate into their practices to mitigate the factors that produce health inequities. ${ }^{2-5}$ The comparable accuracy of POCUS to gold-standard imaging for conditions such as rotator cuff tear, deep vein thrombosis, pleural effusion, and pneumonia prevents barriers such as access to health care, cost, and difficulties obtaining reliable transportation from delaying the diagnosis and treatment of these conditions. ${ }^{2-5}$ Indeed, existing research

This article was externally peer reviewed.

Submitted 27 August 2020; revised 21 January 2021; accepted 23 March 2021.

From the Maxwell Air Force Base, US Air Force, Montgomery, AL (MT).

Funding: None.

Conflict of interest: None.

Prior presentation: This commentary has not been published or presented previously in any format.

Disclaimer: The views expressed are solely those of the author and do not reflect the official policy or position of the US Army, US Navy, US Air Force, the Department of Defense, or the US Government.

Corresponding author: Michael Tanael, MD, Flight Medicine, 300 South Twining Street \#760, Montgomery, AL, 36112, Phone: 808-425-0085 (E-mail: Mtanael@gmail. com). has suggested that POCUS use by primary care clinicians can reduce requirements for formal imaging studies. ${ }^{6,7}$ POCUS also enables outpatient generalists to perform guideline-directed screenings for obstetric abnormalities and aortic abdominal aneurysm (AAA) as well as safely perform procedures such as arthrocentesis; joint injections; and foreign body removal, obviating the need for appointments with specialists - that socioeconomic factors can make difficult to arrange, arrive at, and afford. ${ }^{2-5}$

Admittedly, the narrative reviews advocating for routine use of POCUS in primary care mostly cite studies done in emergency departments and specialty clinics. Reliance on data from these nonprimary care settings raises concern for spectrum bias due to differences in disease severity between disparate patient populations. ${ }^{8}$ However, even if outpatient generalists limit their use of POCUS to applications shown to have acceptable accuracy in a primary care setting, the technology still has the potential to improve health equity substantially.

Two systematic reviews from 2019 identified 8 unique studies assessing the accuracy of POCUS performed by primary care clinicians in a primary care setting. ${ }^{9,10}$ The reviews found 2 studies evaluating primary care clinicians' performance of cardiac exams, 1 evaluating the accuracy of lung examination, 1 evaluating the accuracy of vascular examination, 3 evaluating accuracy of examination for AAA, and 1 evaluating primary care clinicians' ability to perform multiple POCUS applications-assessing identification of AAA, gallstones, ascites, intrauterine pregnancy, and gestational age. All studies showed a favorable comparison of the scans performed by outpatient generalists in a primary care setting with the reference standard, results that suggest primary care 
clinicians can successfully integrate several applications of the technology into their practices.

It seems apparent that if outpatient generalists accurately conduct guideline-directed screening, indicated diagnostic imaging, and procedures under direct visualization in patients who would not otherwise receive such care, then health equity will improve. Unfortunately, as with most interventions that address this problem, little data exists to confirm this. ${ }^{1}$ POCUS does, however, more readily lend itself to study than other proposed technologic interventions aimed at redressing health inequities, such as improving the interoperability of digital platforms. For example, in a patient population plagued by health inequities, a feasible randomized or observational trial could compare endpoints such as time to diagnosis, number of referrals to specialists, percentage of formal imaging studies ordered that reveal clinically significant results, and rates of guideline-directed screenings in patients receiving care from clinicians who have integrated POCUS into their practices and those who have not. Results that favor the group receiving POCUS-enhanced care would support the notion that this technology can mitigate health inequities.

For patients to realize the benefits of POCUS, clinicians must undergo adequate training with the technology. Before scanning, clinicians should develop a basic familiarity with the physics of ultrasound, knobology, and applications of POCUS. They can then establish proficiency in a specific application by satisfactorily performing, interpreting, and integrating the findings of a minimum number of scans (typically 25 to 50 ). ${ }^{11}$ Recommendations further call for a total of 150 to 300 scans (either directly supervised or evaluated through an image portfolio) that include a variety of normal and pathologic findings on patients with a wide range of body types before considering a trainee competent. ${ }^{11}$ While short training courses and experiences in medical school provide excellent foundational knowledge and familiarity with POCUS, the comprehensive nature of current recommendations makes completing an ultrasound curriculum in residency the optimal pathway to achieve competency.

Unfortunately, widespread integration of POCUS training into the residency experience of future primary care clinicians faces many challenges: lack of faculty with adequate POCUS skill, lack of funding, lack of consensus on documenting POCUS exams and receiving reimbursement for them, lack of ACGME requirements to guide POCUS training and ensure its quality, and lack of available time within existing curricula to incorporate it. ${ }^{12,13}$ Unsurprisingly, only a minority of family medicine residencies have a formal POCUS curriculum. ${ }^{12,13}$ However, despite the challenges of implementing a POCUS curriculum into a residency training program, enthusiasm for the technology does seem to be growing, and-undergirded by a belief in the diagnostic and procedural usefulness of POCUS - many residency programs have plans to implement POCUS training into the residency experience. ${ }^{12,13}$ Recognition that POCUS can improve health equity should garner further support for this implementation.

POCUS redresses health inequities by enhancing outpatient generalists' capacity to screen, diagnose, and safely perform procedures in patients who may not otherwise receive such care due to economic, social, educational, health care, and environmental barriers. The body of evidence supporting its use in primary care remains small but promising, and scarcity of data — especially on health outcomes-plagues many proposals to improve health equity, not just POCUS. The potential for POCUS to provide a means for primary care clinicians to redress health inequities should bolster an already growing enthusiasm for the technology and motivate its study, its integration into family medicine training programs, and its uptake by practicing clinicians.

To see this article online, please go to: http://jabfm.org/content/ 34/4/853.full.

\section{References}

1. National Academies of Sciences, Engineering, and Medicine; Health and Medicine Division; Board on Health Care Services; Committee on Integrating Social Needs Care into the Delivery of Health Care to Improve the Nation's Health. Integrating Social Care into the Delivery of Health Care: Moving Upstream to Improve the Nation's Health. Washington (DC): National Academies Press (US);2019.

2. Arnold MJ, Jonas CE, Carter RE. Point-of-care ultrasonography. Am Fam Physician 2020;101:275285.

3. Bornemann P, Jayasekera N, Bergman K, Ramos M, Gerhart J. Point-of-care ultrasound: coming soon to primary care? J Fam Pract 2018;67:70-80.

4. Bornemann P, Barreto T. Point-of-care ultrasonography in family medicine. Am Fam Physician 2018;98:200-202. 
5. Shen-Wagner J, Deutchman M. Point-of-care ultrasound: a practical guide for primary care. Fam Pract Manag 2020;27:33-40.

6. Evangelista A, Galuppo V, Méndez J, et al. Handheld cardiac ultrasound screening performed by family doctors with remote expert support interpretation. Heart 2016;102:376-382.

7. Colli A, Prati D, Fraquelli M, et al. The use of a pocket-sized ultrasound device improves physical examination: results of an in- and outpatient cohort study. PLoS One 2015;10:e0122181.

8. Guyatt G, Rennie D, Meade MO, Cook DJ. Users' Guides to the Medical Literature: Essentials of EvidenceBased Clincial Practice. 3rd ed. New York: McGrawHill Medical;2015.

9. Andersen CA, Holden S, Vela J, et al. Point-of-care ultrasound in general practice: a systematic review. Ann Fam Med 2019;17:61-69.
10. Sorensen B, Hunskaar S. Point-of-care ultrasound in primary care: a systematic review of generalist performed point-of-care ultrasound in unselected populations. Ultrasound J 2019;11:31.

11. American Academy of Family Physicians. Family Medicine Residency Curriculum Guidelines: Pointof-Care ultrasound. Available from: https://www.aafp. org/dam/AAFP/documents/medical_education_ residency/program_directors/Reprint290D_POCUS. pdf. Published Dec 2016. Accessed December 23, 2020.

12. Hall JW, Holman H, Barreto TW, et al. Pointof-care ultrasound in family medicine residencies 5-year update: a CERA study. Fam Med 2020;52:505-511.

13. Micks T, Braganza D, Peng S, et al. Canadian national survey of point-of-care ultrasound training in family medicine residency programs. Can Fam Physician 2018;64:e462-e467. 